Supporting Information

\title{
Multifunctional injectable hydrogel for in vivo diagnostic and therapeutic applications
}

Gi Doo Cha ${ }^{1,2 \perp}$, Wang Hee Lee $e^{1,2 \perp}$, Sung-Hyuk Sunwoo ${ }^{1,2 \perp}$, Dayoung Kang ${ }^{1,2}$, Taegyu Kang ${ }^{1,2}$, Kyoung Won Cho ${ }^{1,2}$, Minjeong Kim ${ }^{1,2}$, Ok Kyu Park ${ }^{1,3}$, Dongjun Jung $^{1,2}$, Jongha Lee ${ }^{1,2}$, Seung Hong Choi ${ }^{1,3 *}$, Taeghwan Hyeon ${ }^{1,2 *}$, Dae-Hyeong $\operatorname{Kim}^{1,2,4 *}$

${ }^{1}$ Center for Nanoparticle Research, Institute for Basic Science (IBS), Seoul 08826, Republic of Korea.

${ }^{2}$ School of Chemical and Biological Engineering, Institute of Chemical Processes, Seoul National University, Seoul 08826, Republic of Korea.

${ }^{3}$ Department of Radiology, Seoul National University College of Medicine, Seoul 03080, Republic of Korea.

${ }^{4}$ Department of Materials Science and Engineering, Seoul National University, Seoul 08826, Republic of Korea.

${ }^{\perp}$ G. D. Cha, W. H. Lee, and S.-H. Sunwoo contributed equally to this work.

*To whom correspondences should be addressed.

E-mail:dkim98@snu.ac.kr,thyeon@snu.ac.kr, and verocay@snuh.org 


\section{Supporting experimental section}

\section{Ex vivo tests}

The interface between the hydrogel and the porcine skin was observed through cryogenictemperature scanning electron microscopy using the Quanta 3D FEG (FEI Company, USA) installed at Korea Institute of Science and Technology. Various hydrogel samples (e.g., pristine hydrogel, hydrogel with Evans blue, and hydrogel with NPs) were injected onto the porcine skin. After gelation, the hydrogels were deformed mechanically by deforming the porcine skin and were then observed.

\section{Synthesis of PLGA nanoparticles}

PLGA NPs were synthesized using the double-emulsion solvent evaporation technique reported in the previous study. ${ }^{1}$ PLGA was dissolved in ethyl acetate $(30 \mathrm{mg} / \mathrm{mL})$, whereas pluronic ${ }^{\circledR} \mathrm{F}-127$ (various concentrations) and the mock-up drug (e.g., fluorescein) $(1 \mathrm{mg} / \mathrm{mL})$ were dissolved in DI water. They were mixed and dispersed by subjecting the solutions to sonication for $2 \mathrm{~min}$. The mixed solution was poured into a sucrose solution $(0.2 \mathrm{~g} / \mathrm{mL})$ and subjected to sonication for $2 \mathrm{~min}$. The final emulsion was stirred overnight to allow evaporation of the solvent and was centrifuged at $10,000 \mathrm{rpm}$ for $10 \mathrm{~min}$ to separate the NPs from the solution. The supernatant was discarded, and the precipitated NPs were redispersed in DI water. The size of the PLGA NPs was varied by changing the amount of surfactant (i.e., pluronic ${ }^{\circledR} \mathrm{F}-$ 127).

\section{Synthesis of FIONs}

FIONs were synthesized using the method reported in the previous study. ${ }^{2}$ Iron(III) acetylacetonate, oleic acid, 4-biphenylcarboxylic acid, and benzyl ether were mixed, and the solution was heated to $290{ }^{\circ} \mathrm{C}$ for 30 mins. Subsequently, the mixture was cooled to room 
temperature, and acetone was added to lower the solubility of the solution. The solution was centrifuged at $1700 \mathrm{rpm}$ for $10 \mathrm{~min}$ to precipitate the NPs, which were redispersed in chloroform. The resulting FIONs in chloroform were mixed with phospholipid-PEG to confer biocompatibility and hydrophilicity to the NPs. Chloroform was evaporated in a vacuum at $80{ }^{\circ} \mathrm{C}$ for $2 \mathrm{~h}$. Subsequently, DI water was added, and sonication was used to disperse the NPs. To remove the excess phospholipid-PEG, the NPs were washed multiple times through ultracentrifugation and size-exclusion chromatography using a Sephadex G-25, PD-10 column.

\section{Protocols for in vivo experiments}

Eight-week-old Sprague Dawley (SD) rats (Orient Bio, Republic of Korea) were anesthetized using 4\% isoflurane (Terrell; Hana Pharm Co., Ltd., Korea) mixed with oxygen and then fixed on an operating table. In some termination experiments, anesthesia was used along with urethane (Sigma Aldrich, $1000 \mathrm{mg} / \mathrm{kg}$ ). 


\section{Supplementary table and figures}

\begin{tabular}{|c|c|c|c|c|c|c|}
\hline Backbone polymer & Injectability & Bioresorbability & Adhesiveness & Stretchability & Remark & Reference \\
\hline Multifunctional hydrogel & o & 0 & 0 & 0 & & Current work \\
\hline PGA-Dopa & 0 & 0 & 0 & $\mathrm{x}$ & & [4] \\
\hline HA/Pluronic F-127 & 0 & 0 & 0 & $x$ & & [5] \\
\hline Alginate & 0 & $\mathrm{x}$ & 0 & 0 & Boronic acid & [6] \\
\hline HA/Glutamic acid & o & o & $\Delta$ & $\Delta$ & & [7] \\
\hline CTS/Pluronic F-127 & o & $\Delta$ & $\Delta$ & $\Delta$ & & [8] \\
\hline GELDA/HA/Ppy-Dopa & 0 & $\Delta$ & 0 & $\mathrm{x}$ & & [9] \\
\hline PGS-PEG-CA/GEL & 0 & 0 & $\Delta$ & $x$ & & [10] \\
\hline Dex/PL-Dopa & 0 & 0 & $\Delta$ & $x$ & & [11] \\
\hline Alg/GEL & 0 & 0 & $\Delta$ & $\mathrm{x}$ & & [12] \\
\hline BAS protein & 0 & 0 & $\mathrm{x}$ & $\Delta$ & & [13] \\
\hline PEGDA/PEG & $\mathrm{x}$ & $\Delta$ & 0 & 0 & & [14] \\
\hline CTS-CA/CTS-MA & $\Delta$ & 0 & 0 & $\mathrm{x}$ & & [15] \\
\hline PACG-Hap & $\mathrm{x}$ & $\Delta$ & 0 & 0 & & [16] \\
\hline CTS/PEO-PPO-PEO & o & $\Delta$ & $\Delta$ & $\Delta$ & & [17] \\
\hline CMC/Cellulose & 0 & $\Delta$ & $\Delta$ & $x$ & & [18] \\
\hline Material from Andrias davidianus & $\mathrm{x}$ & 0 & 0 & $\mathrm{x}$ & & [19] \\
\hline PAM & $\mathrm{x}$ & $\mathrm{x}$ & o & 0 & & [20] \\
\hline Silk Fibrion & $x$ & 0 & o & $x$ & & [21] \\
\hline HA/CTS & $\mathrm{x}$ & o & o & $\mathrm{x}$ & & [22] \\
\hline Catechol-functional elastin-like polypeptides & $\mathrm{x}$ & 0 & 0 & $\mathrm{x}$ & & [23] \\
\hline HA/CTS & $\mathrm{x}$ & 0 & $\mathrm{x}$ & 0 & & [24] \\
\hline CTS/PEG & o & $\Delta$ & $\Delta$ & $\mathrm{x}$ & & [25] \\
\hline HA-NB & $\Delta$ & $\mathrm{x}$ & 0 & $\mathrm{x}$ & Toxic photoinitiator & [26] \\
\hline PAM/GEL & $\Delta$ & $\mathrm{x}$ & $\mathrm{x}$ & 0 & & [27] \\
\hline GEL/PAC & $\Delta$ & $\mathrm{x}$ & $\mathrm{x}$ & 0 & & [28] \\
\hline GEL-Dopa & o & $\mathrm{x}$ & $\Delta$ & $\mathrm{x}$ & Toxic crosslinker & [29] \\
\hline PEG & 0 & $\Delta$ & $\mathrm{x}$ & $\mathrm{x}$ & & [30] \\
\hline PEG-PCL-PEG & o & $\Delta$ & $\mathrm{x}$ & $\mathrm{x}$ & & [31] \\
\hline CTS/GEL & 0 & $\Delta$ & $\mathrm{x}$ & $\mathrm{x}$ & & [32] \\
\hline PAM & $\mathrm{x}$ & $\mathrm{x}$ & $\Delta$ & 0 & & [33] \\
\hline PAM & $\mathrm{x}$ & $\mathrm{x}$ & $\Delta$ & 0 & & [34] \\
\hline poly(dopamine-co-acrylate) & $\mathrm{x}$ & $\Delta$ & 0 & $\mathrm{x}$ & & [35] \\
\hline PEGDA & $\mathrm{x}$ & $\Delta$ & 0 & $\mathrm{x}$ & & [36] \\
\hline PEG-Dopa & $\mathrm{x}$ & $\Delta$ & 0 & $\mathrm{x}$ & & [37] \\
\hline PEG & $\mathrm{x}$ & $\Delta$ & $\Delta$ & $\mathrm{x}$ & & [38] \\
\hline GELMA/PEGDA & $\mathrm{x}$ & $\mathrm{x}$ & 0 & $\mathrm{x}$ & Toxic Acrylic acid & [39] \\
\hline GEL/Alg & $\mathrm{x}$ & $\mathrm{x}$ & 0 & $\mathrm{x}$ & Toxic EDC & [40] \\
\hline PAM/Alg & $\mathrm{x}$ & $\mathrm{x}$ & $\mathrm{x}$ & 0 & & [41] \\
\hline GELMA & $\Delta$ & $\Delta$ & $\mathrm{x}$ & $\mathrm{x}$ & & [42] \\
\hline poly(dimethyl diallyl ammonium chloride) & $\mathrm{x}$ & $\mathrm{x}$ & 0 & $x$ & & [43] \\
\hline PAM/HAP & $\Delta$ & $\mathrm{x}$ & $\mathrm{x}$ & $\mathrm{x}$ & & [44] \\
\hline
\end{tabular}

Table S1. Comparison of key material properties of various hydrogels. Many hydrogels were compared according to the degree of injectability, bioresorbability, adhesiveness, and stretchability. The criteria for evaluation are as follows: (i) Injectability: $\mathbf{O}$ injectable without the aid of external energetic stimuli, $\triangle$ injectable with the aid of external energetic stimuli (e.g., UV), $\times$ not injectable; (ii) Bioresrobability: $\mathbf{O}$ natural bioresorbable polymer, $\triangle$ biocompatible but non-degradable polymer composed of biocompatible monomers, $\times$ non-biodegradable polymer composed of toxic monomers or synthesized with toxic additives; (iii) Adhesiveness: $\mathbf{O}$ adhesion strength higher than that of a commercial bio-glue ( $20 \mathrm{kPa}$; Figure $2 \mathrm{f}), \triangle$ adhesion strength lower than that of a commercial bio-glue, $\times$ no adhesion; (iv) Stretchability: $\mathbf{O}$ 
stretchability higher than that of bladder $(\lambda=1.8),{ }^{3} \triangle$ stretchability smaller than that of bladder, $X$ no stretchability. The searched references are arranged in order of high evaluation. ${ }^{4-44}$ 
a
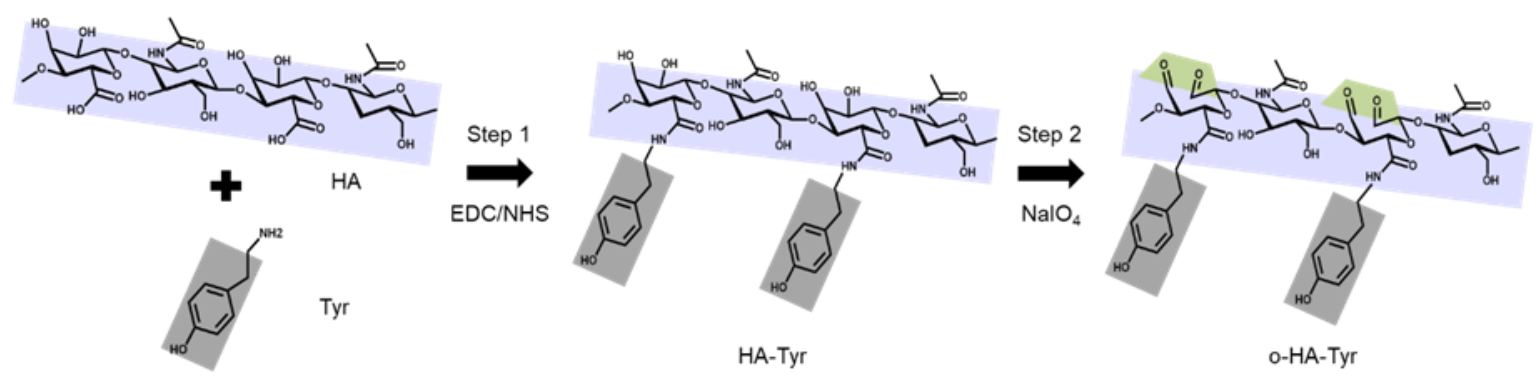

b
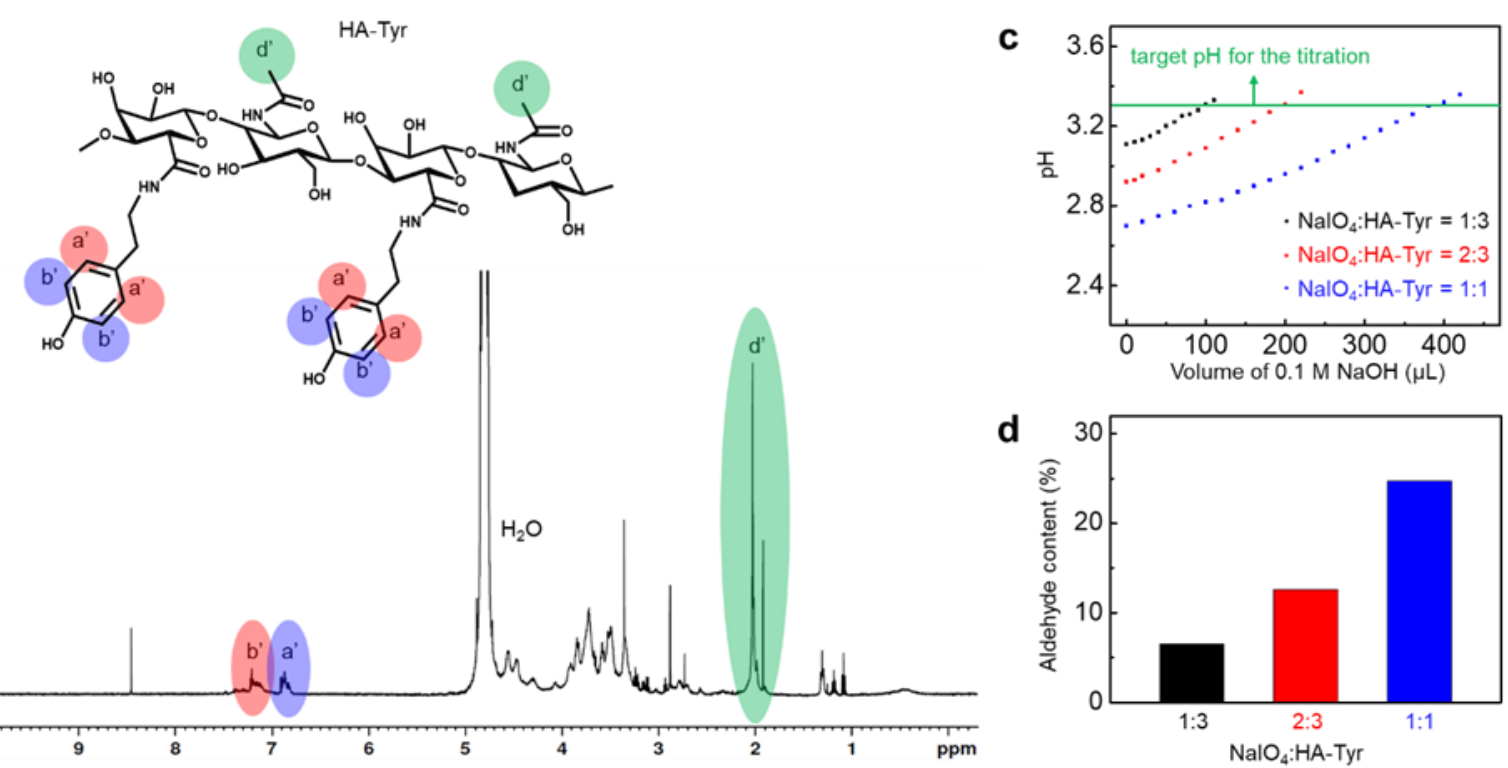

Figure S1. Synthesis and characterization of o-HA-Tyr. (a) Two-step synthesis of o-HA-Tyr. HA-Tyr was synthesized using the EDC/NHS coupling agent, and it was oxidized by $\mathrm{NaIO}_{4}$. The synthesized o-HA-Tyr is refined through the dialysis process to separate the toxic coupling/oxidizing agents completely. (b) ${ }^{1} \mathrm{H}$ NMR spectroscopy analysis to estimate the conjugation ratio of HA-Tyr. The conjugation ratio was 30\%. (c) The amount of aldehyde was measured by titration of o-HA-Tyr with $0.1 \mathrm{M} \mathrm{NaOH}$. Three kinds of samples (1:3, 2:3, and 1:1; molar ratio of $\mathrm{NaIO}_{4}$ and $\mathrm{HA}-\mathrm{Tyr}$ ) were used for the titration. (d) The amount of aldehyde in o-HA-Tyr (or degree of oxidation) increases as the fraction of $\mathrm{NaIO}_{4}$ in the mixture of $\mathrm{NaIO}_{4}$ and HA-Tyr increases. For the multifunctional injectable hydrogel, o-HA-Tyr oxidized by a molar ratio of 1:1 ( $\left.\mathrm{NaIO}_{4}: \mathrm{HA}-\mathrm{Tyr}\right)$ was employed due to its high aldehyde amount $(\sim 25 \%)$. 


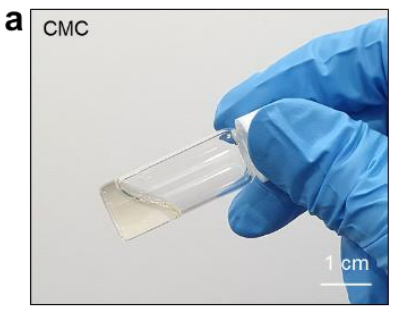

$a+c$
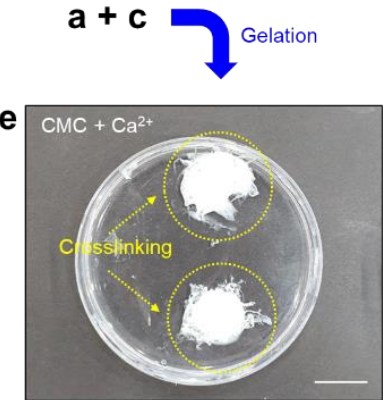

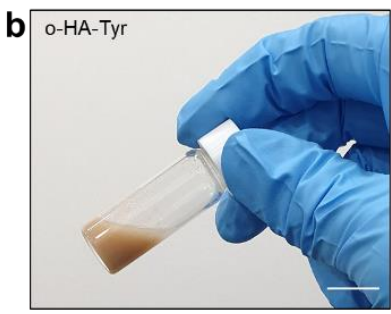

$\mathbf{b}+\mathbf{d}$
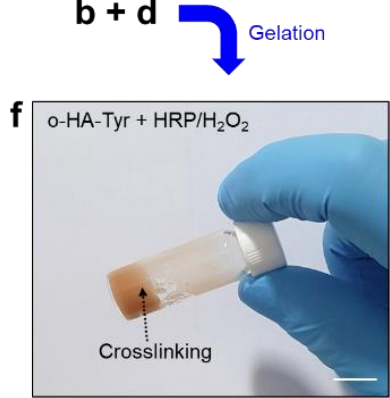

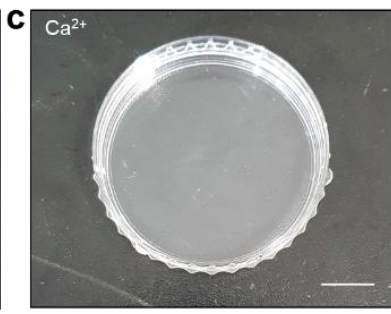

$a+d$
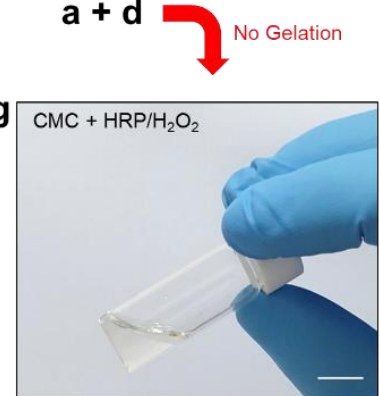

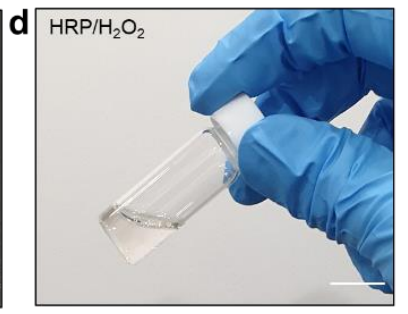

$\mathbf{b}+\mathbf{c}$ No Gelation

Figure S2. Schematic illustrations for the enzymatic and ionic crosslinking reaction and images for the split test to confirm the reaction selectivity. Ionic crosslinking of CMC mediated by $\mathrm{Ca}^{2+}$. Enzymatic crosslinking of o-HA-Tyr mediated by HRP and $\mathrm{H}_{2} \mathrm{O}_{2}$. (a-d) A series of split tests confirmed the reaction selectivity of the ionic and enzymatic crosslinking. The solutions of the polymer (a) CMC and (b) o-HA-Tyr) and those of the crosslinking mediator (c) $\mathrm{Ca}^{2+}$ and (d) $\mathrm{HRP} / \mathrm{H}_{2} \mathrm{O}_{2}$ ) were prepared. (e-h) The polymer and the crosslinking mediator were mixed with each other. The crosslinking of (e) $\mathrm{CMC}$ with $\mathrm{Ca}^{2+}(\mathrm{a}+\mathrm{c})$ and (f) o-HA-Tyr with $\mathrm{HRP} / \mathrm{H}_{2} \mathrm{O}_{2}(\mathrm{~b}+\mathrm{d})$ could be visually confirmed. However, (g) CMC with $\mathrm{HRP} / \mathrm{H}_{2} \mathrm{O}_{2}(\mathrm{a}+\mathrm{d})$ and (h) o-HA-Tyr with $\mathrm{Ca}^{2+}(\mathrm{b}+\mathrm{c})$ showed no clue for the crosslinking. 

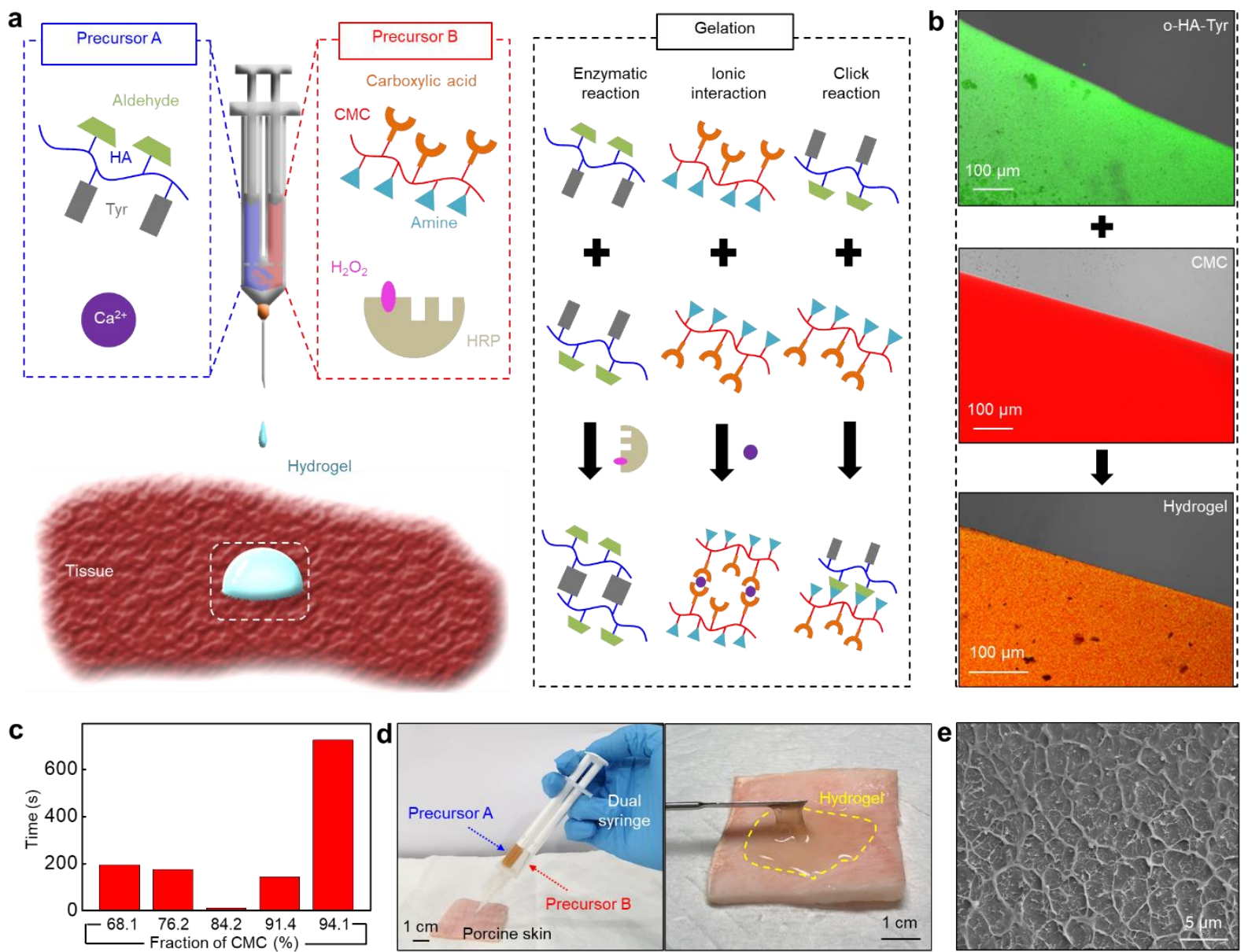

Figure S3. Injectability of the hydrogel. (a) As two separated precursors are mixed by their injection through a dual syringe, multiple reactions occur spontaneously, which leads to the insitu solidification of the hydrogel. (b) The homogeneous mixing of precursors was examined by confocal microscopy. Precursors (including dyes for visualization) were imaged (o-HA-Tyr with fluorescein (top), CMC with rhodamine (middle)), and the hydrogel obtained by mixing them was also imaged (bottom). The uniform color change confirms the homogeneous mixing. (c) Gelation time of the hydrogel with various fractions of CMC. (d) The injectability of the precursors (left) and the resulting hydrogel (right) was visually confirmed on the porcine skin ex vivo. (e) Cryo-scanning electron microscope image of the hydrogel. 


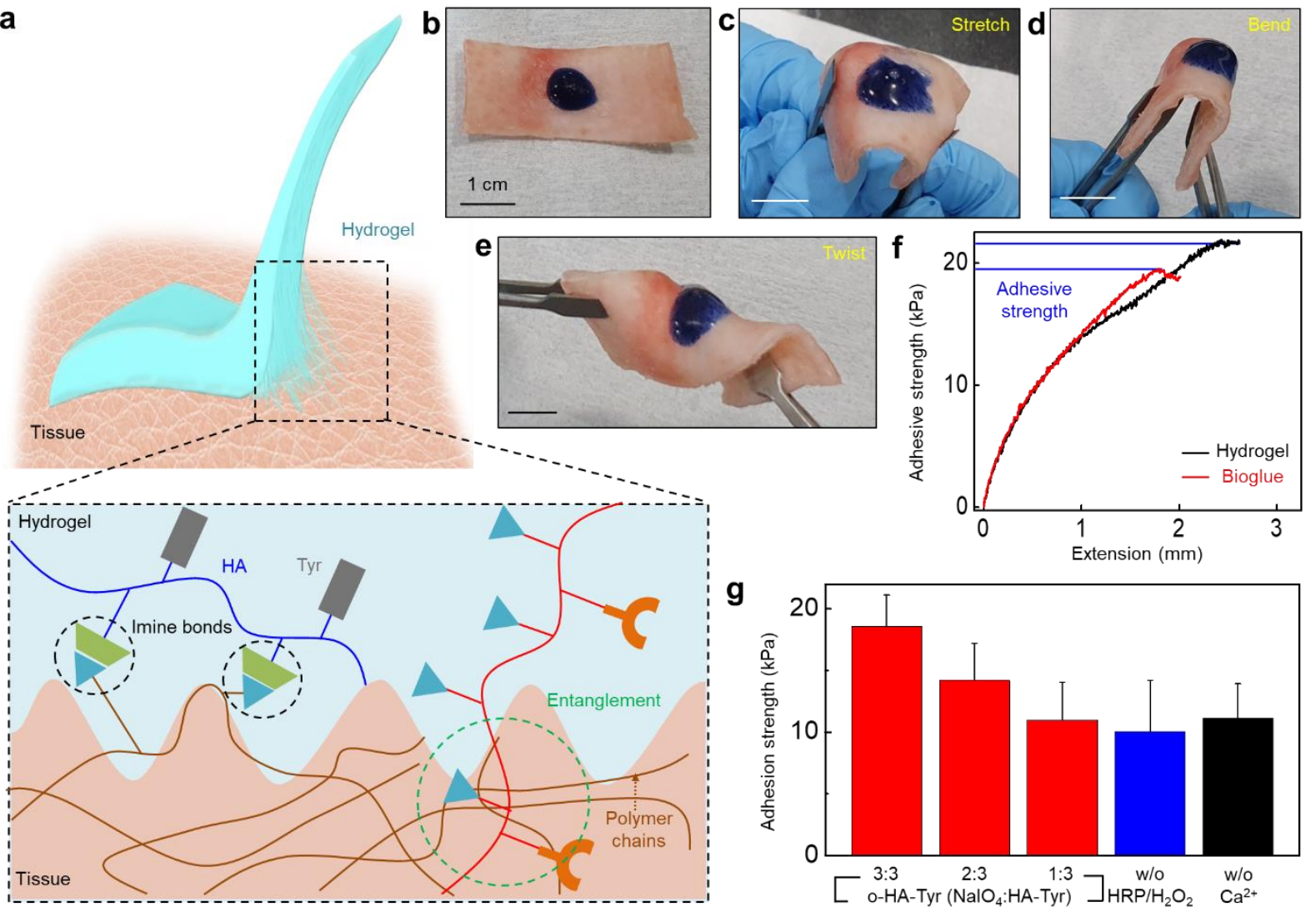

Figure S4. Adhesiveness of the hydrogel. (a) Two major factors (chemical bonds and entanglement of polymer chains) contribute to the adhesion of the hydrogel to the tissue surface. ${ }^{45}$ One is the chemical bond between the aldehyde group of o-HA-Tyr in the hydrogel and the amine moieties in the tissue. Another is the entanglement between the polymer chain of the hydrogel and the tissue. (b-e) The strong adhesion of the hydrogel was examined by deforming the tissue, on which the hydrogel is attached. (b) The hydrogel was dyed with Evans blue for its visualization on the porcine skin. The hydrogel maintained a good adhesion to the tissue surface despite various deformations, including (c) stretching, (d) bending, and (e) twisting. (f) To quantify the adhesion strength, lap-shear tests for the hydrogel (black curve) and the control group (bioglue; red curve) were conducted. The adhesion strength was determined as the highest value during the detachment of the sample from the porcine skin tissue. (g) Adhesion strengths of the hydrogel with various degrees of oxidation of o-HA-Tyr on bare tissue (red bars). In the case of hydrogels composed of $91.4 \% \mathrm{CMC}$ without $\mathrm{HRP} / \mathrm{H}_{2} \mathrm{O}_{2}$ (blue bar) or $91.4 \% \mathrm{CMC}$ without $\mathrm{Ca}^{2+}$ (black bar), the adhesion becomes poor due to less crosslinking and entanglement of polymer chains. 
a
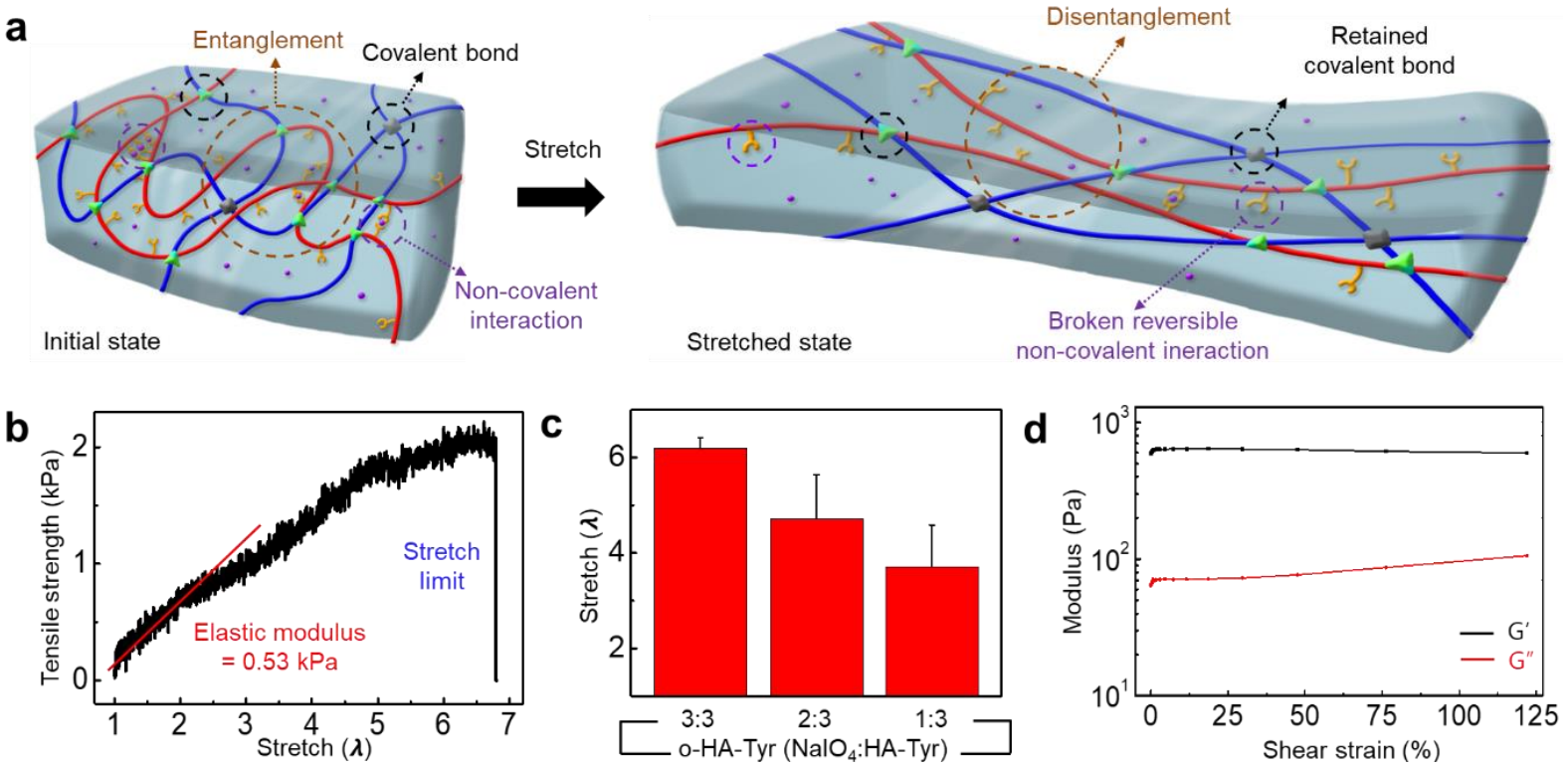

Figure S5. Stretchability of the hydrogel. (a) Multiple reactions (black or purple dotted circle) and entanglement (brown dotted circle) between backbone polymers result in the soft doublenetwork hydrogel. In detail, o-HA-Tyr (blue line) consists of a chemically crosslinked network formed by a strong irreversible reaction (grey square in a black dotted circle), while CMC (red line) consists of a physically crosslinked network formed by a weak reversible interaction (orange tongs in the purple dotted circle), and these two polymer chains are crosslinked and entangled each other through a chemical bond (green triangle in a black dotted circle). The coexistence of weak and strong bonds has the advantage of enhancing the mechanical property of the hydrogel. ${ }^{37}$ When the hydrogel is stretched, the non-covalent interaction and the entanglement between polymers are broken and unraveled transiently to endure the external mechanical deformation, while the covalent bond maintains the structure of the hydrogel. (b) Stretchability of the hydrogel was determined when the tensile strength reached the highest value. The elastic modulus of the hydrogel is under $1 \mathrm{kPa}$, which shows the softness of the hydrogel. (c) Stretchability of the hydrogel increases as the degree of oxidation of o-HA-Tyr increases. (d) Strain-dependent shear rheology of the hydrogel indicates that the hydrogel is stable under the external strain up to $120 \%$. 

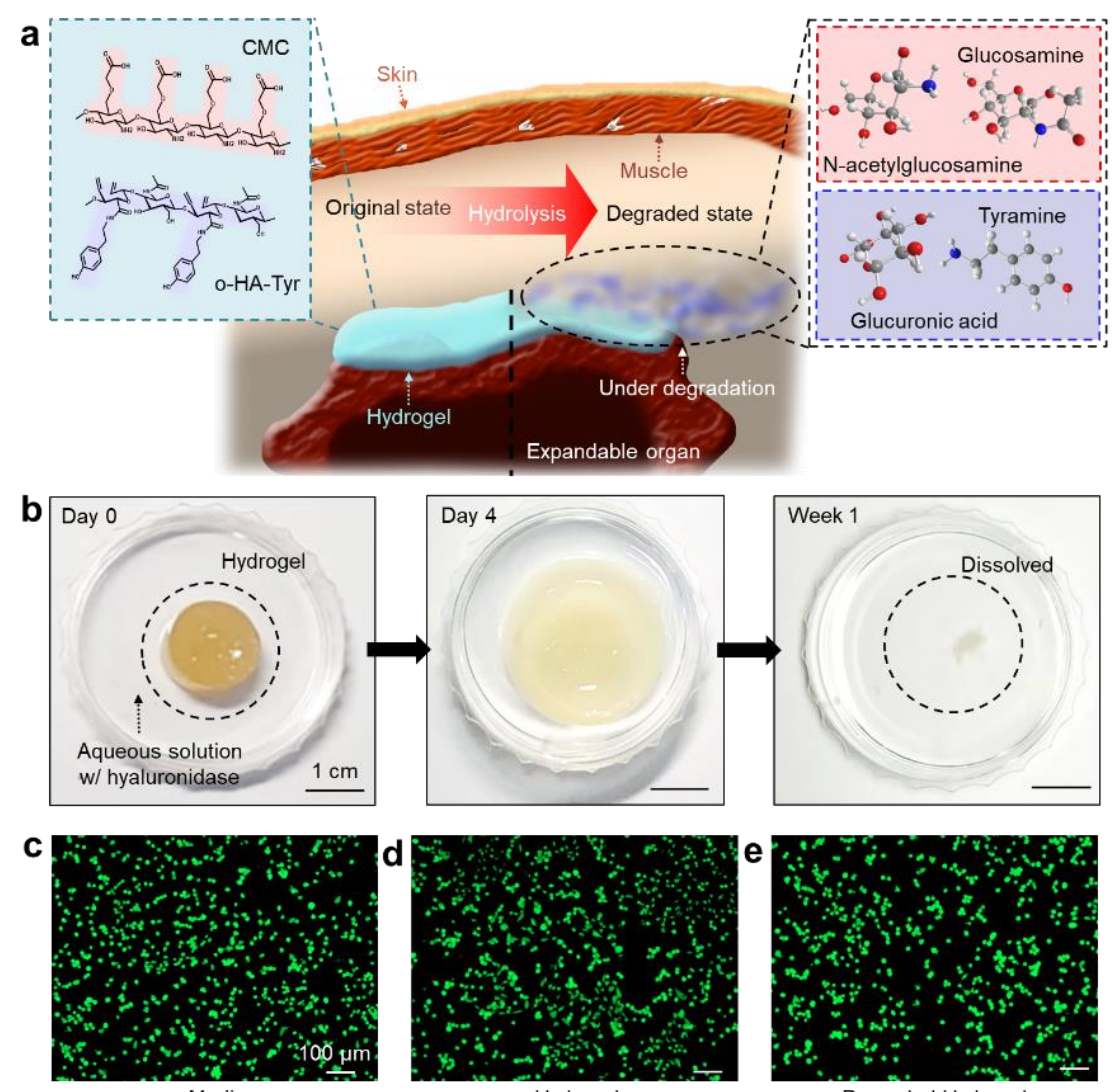

Media

Hydrogel

Degraded Hydrogel
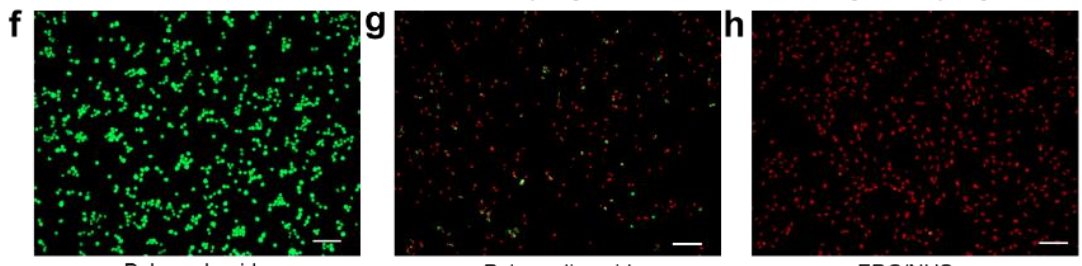

Polyacrylamide

Polyacrylic acid

EDC/NHS
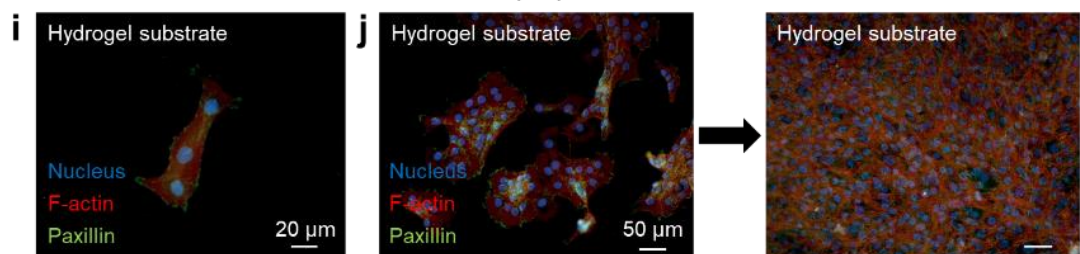

Figure S6. Bioresorbability of the hydrogel. (a) The hydrogel consists of natural biopolymers that can be decomposed into metabolites in vivo. $\mathrm{CMC}$ is decomposed into $\mathrm{N}$ acetylglucosamine and glucosamine, and o-HA-Tyr is decomposed into tyramine and glucuronic acid. (b) The hydrogel can be decomposed in an aqueous solution of hyaluronidase in a week. (c-h) The Live/Dead cell viability assay of the indicated groups (group: (c) media, (d) hydrogel, (e) degraded hydrogel, (f) polyacrylamide, (g) polyacrylic acid, (h) EDC/NHS) with $20 \mathrm{mM}$ concentration. The hydrogel, degraded hydrogel, and PAM did not show any severe cytotoxicity regardless of the concentration. Pac showed some extent of toxicity, and EDC/NHS showed severe cytotoxicity. These results are consistent with the MTT cell viability assay in Figure $4 \mathrm{j}$. The degraded hydrogel was a solution that the hydrogel soaked in a cell medium 1 week before the MTT test. (i) Fluorescent analysis of C2C12 cells cultured on the hydrogel substrate. The nucleus is stained in blue, F-actin in red, and myotube in green. (j) Fluorescent analysis of the proliferation of $\mathrm{C} 2 \mathrm{C} 12$ cells cultured on the hydrogel substrate. The nucleus is stained in blue, F-actin in red, and Paxillin in green. 


\section{a}

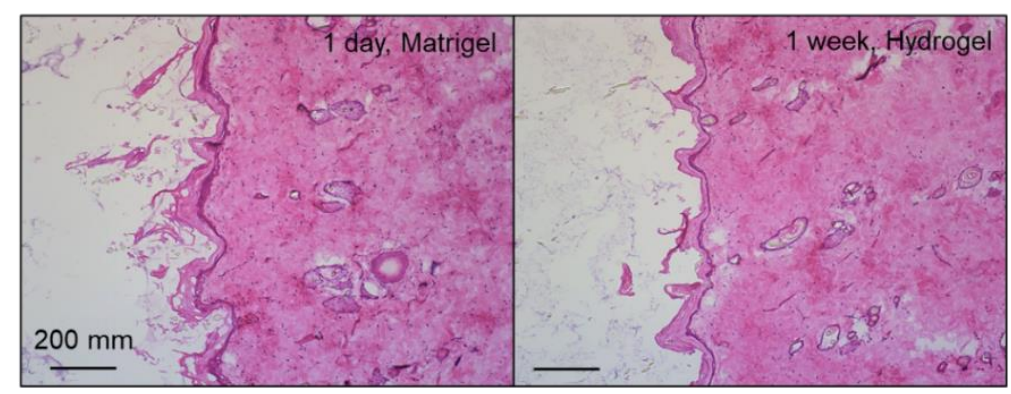

b

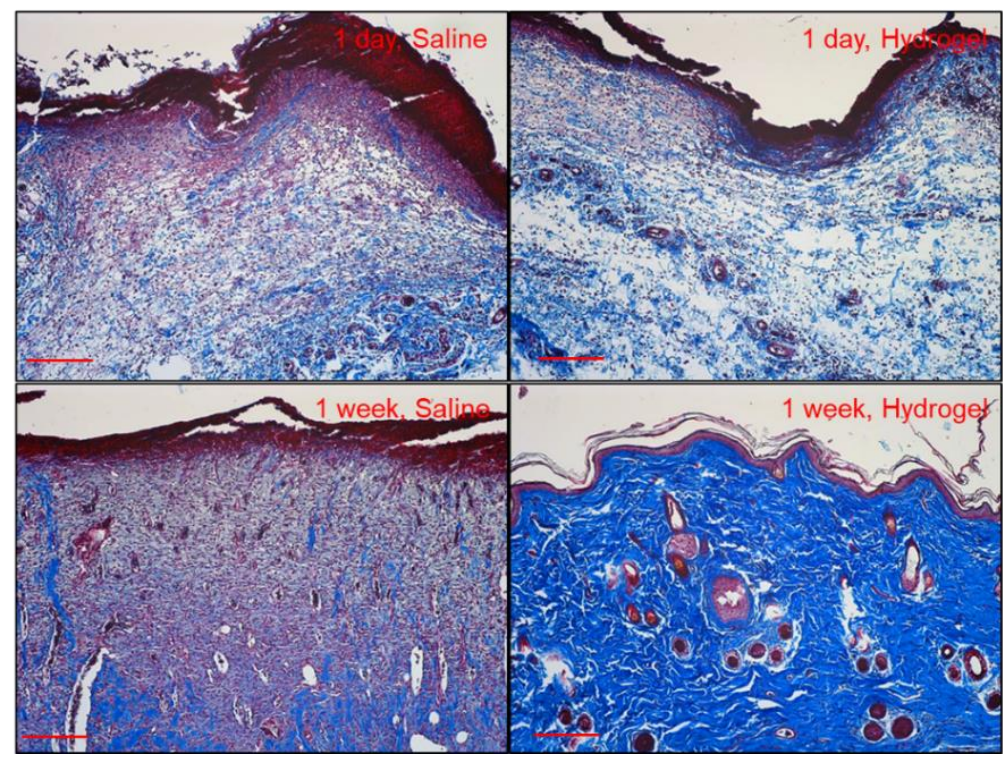

Figure S7. Histochemical staining of the tissue after application of the hydrogel (Matrigel). (a) Histological analysis (H\&E staining) of the tissues near the implanted Matrigel at different time points (1 day and 1 week). (b) Histological analysis (Masson's trichrome staining) of the wounded tissues after application of the saline and the hydrogel at different time points (1 day and 1 week). The saline solution was used as a control group. 

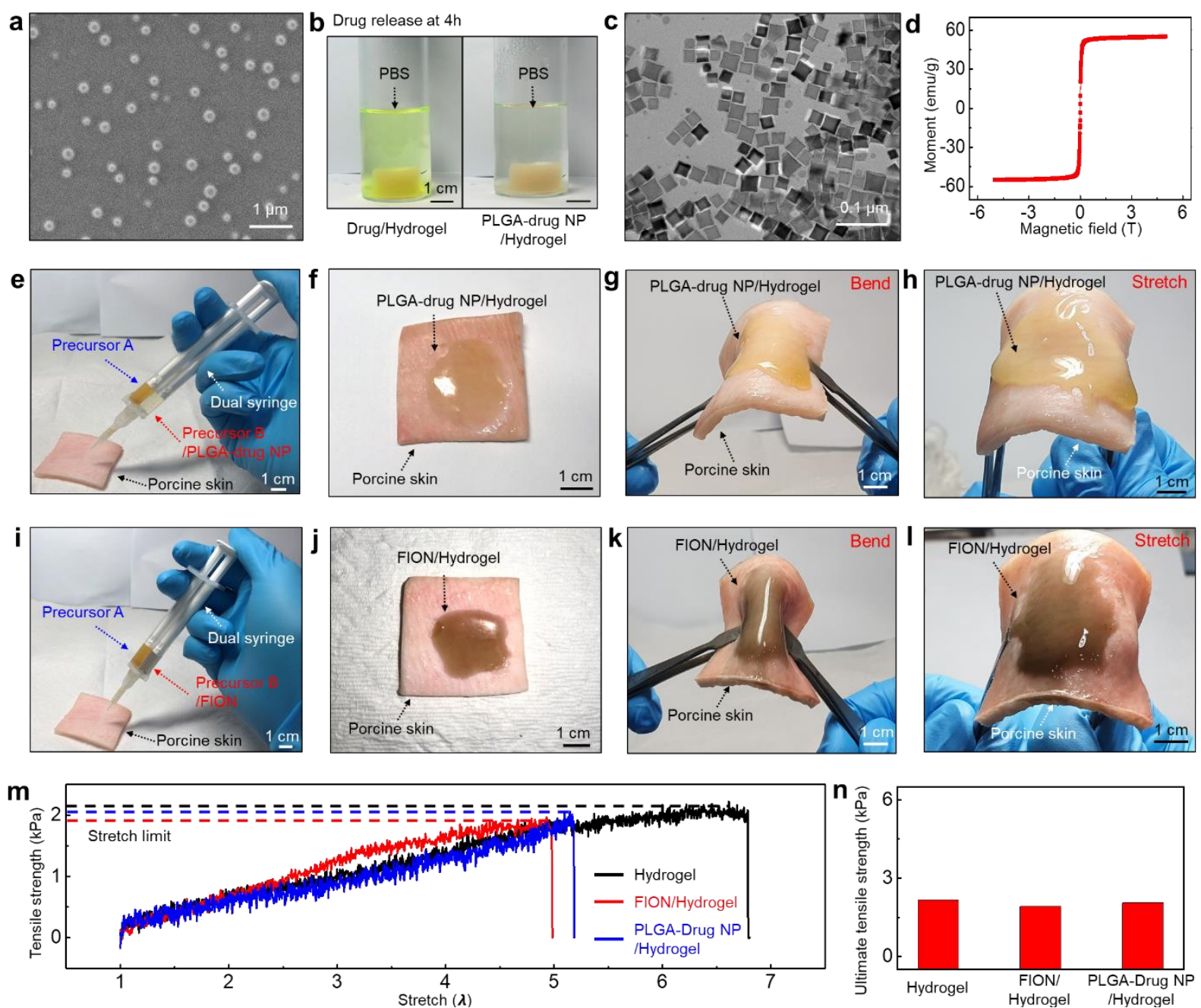

Figure S8. Hydrogel nanocomposites with drug-loaded PLGA NPs and FIONs. (a) Scanning electron microscope image of PLGA-drug NPs. (b) Optical camera image of the Drug/Hydrogel composite (left) and the PLGA-drug NPs/Hydrogel composite (right) soaked in phosphate-buffered saline (PBS) solution for 4 hours at $37^{\circ} \mathrm{C}$. (c) Transmission electron microscope image of FIONs. (d) Magnetic hysteresis loop of FIONs measured at $300 \mathrm{~K}$. (e) The precursors containing PLGA-drug NPs were injected and (f) the hydrogel nanocomposite was successfully formed on the porcine skin. The PLGA-drug NP/Hydrogel composite maintains its structure and good adhesion to the tissue despite various mechanical deformations such as (g) bending and (h) stretching of the porcine skin. (i) The precursors containing FIONs were injected and (j) the hydrogel nanocomposite was successfully formed on the porcine skin. The FIONs/Hydrogel composite maintains its structure and good adhesion to the tissue despite various mechanical deformations such as (k) bending and (l) stretching of the porcine skin. (m) The ultimate tensile strength of the hydrogel, FION/Hydrogel nanocomposite, and PLGA-Drug $\mathrm{NP} / \mathrm{Hydrogel}$ nanocomposite was determined when the tensile strength reached the highest value. (n) The ultimate tensile strength of the hydrogel, FION/Hydrogel nanocomposite, and PLGA-Drug NP/Hydrogel nanocomposite. 


\section{Captured images from Supplementary Movies}
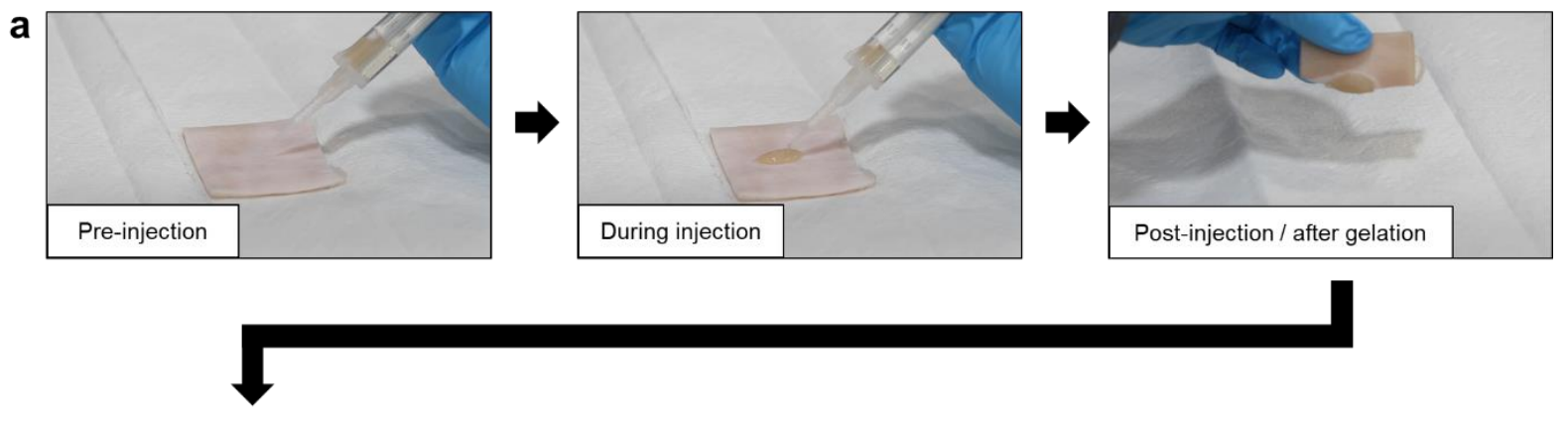

b
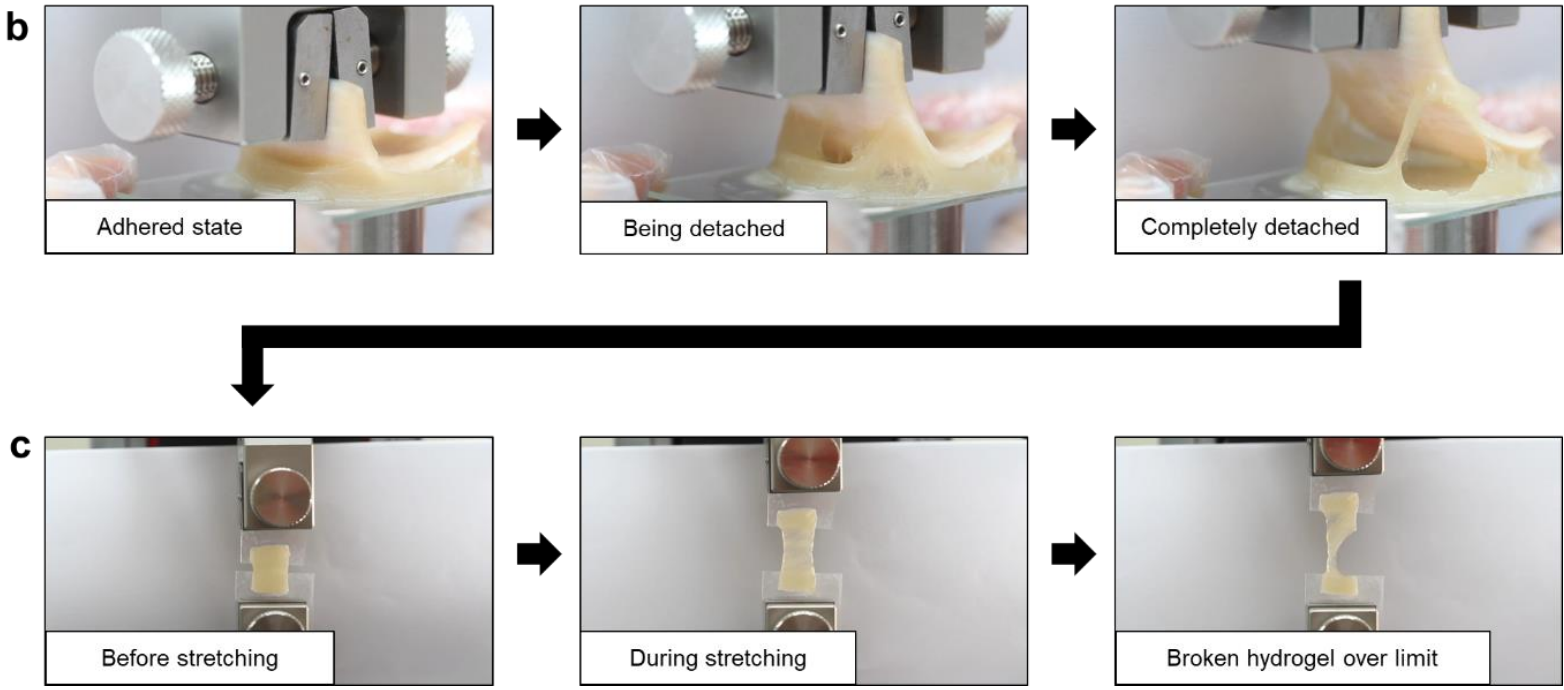

Movie S1. Feasibility test using porcine skin ex vivo. Images were captured from Movie S1 to show its key points. A full Supporting Movie is available for download. The design strategy of the multifunctional hydrogel was examined through a simple feasibility test. (a) The hydrogel precursors were injected on the surface of the porcine skin ex vivo. In a few minutes, the spontaneous gelation occurred without external energetic stimuli. To confirm the complete gelation of the hydrogel, the porcine tissue was bent and turned upside down. (b) The hydrogel made a strong adhesion to the porcine skin. To confirm this strong adhesion, the porcine skin was pulled upward to be detached from the hydrogel. For this 90-degree peel test, the hydrogel was fixed on the glass substrate. The elongation of the hydrogel could be observed during the detachment procedure. (c) The hydrogel is highly stretchable. To test the stretchability, PET films attached to both ends of the hydrogel were pulled uniaxially to the point of the elongation at break. When the elongation is over the maximum stretchability $(\lambda=6.6)$, the crack propagates from the edge to the center of the hydrogel, and eventually, the hydrogel breaks down into two pieces. 


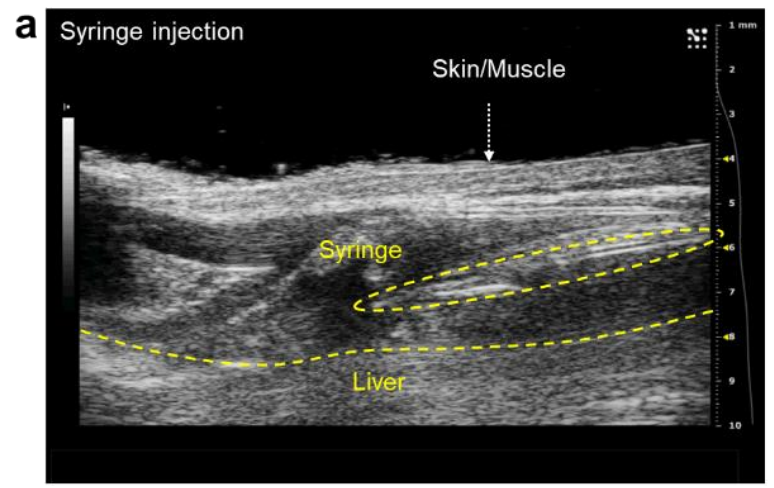

Before the injection of precursors from the syringe
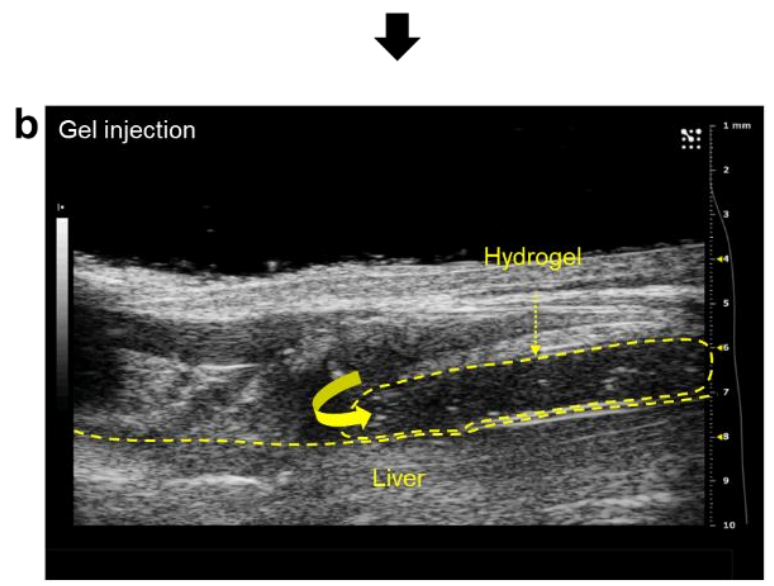

The injection of precursors
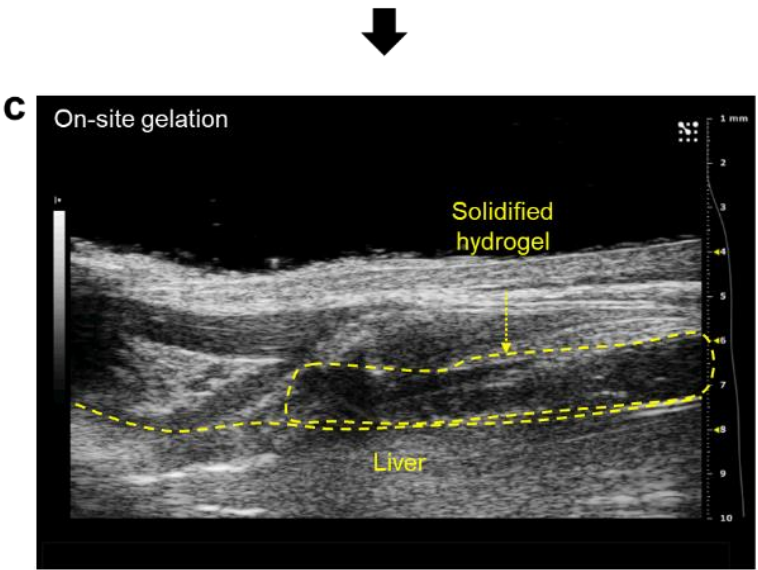

The solidified hydrogel after in-situ gelation of precursors

Movie S2. Real-time observation of the injection of precursors onto a rat's liver and the solidification of the hydrogel in vivo by ultrasound imaging. Images were captured from Movie S2 to show its key points. A full Supporting Movie is available for download. Movie S2 was taken by using an Ultrasound system (Vevo2010, FUJIFILM VisualSonics Inc., Japan) and a probe (Lz-550, $40 \mathrm{MHz}$, B-Mode). Ultrasound imaging showed the process of precursor injection and the in-situ gelation of the hydrogel on the liver of a rat (a) before the injection of precursors from the syringe, (b) after the injection of precursors, and (c) after the in-situ solidification of the hydrogel. 


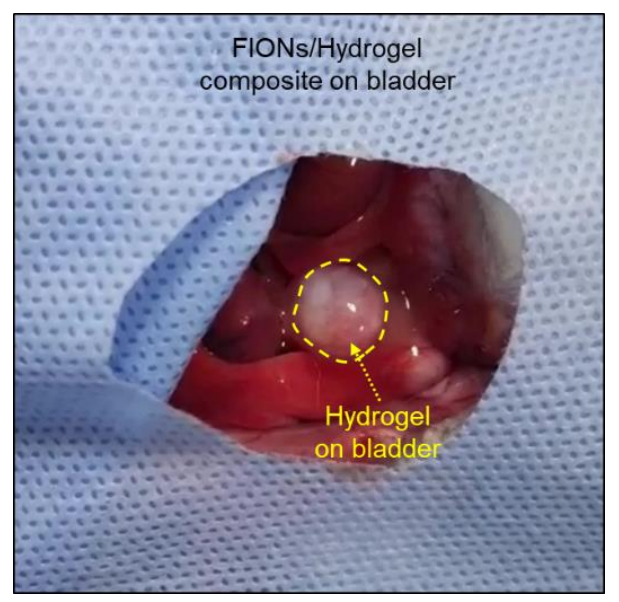

Movie S3. Application of the hydrogel nanocomposite with FIONs onto the surface of the bladder in vivo. Images were captured from Movie S3 to show its key points. A full Supporting Movie is available for download. The hydrogel nanocomposite with FIONs was applied on the surface of the urinary bladder, and the bladder was expanded by injecting the PBS solution into the bladder. The hydrogel nanocomposite maintained its firm adhesion with the bladder regardless of the dynamic volume change of the bladder due to its strong adhesion and high stretchability. More detailed snapshot images are shown in Figure 5m. 


\section{References}

(1) Ramalho, M. J.; Pereira, M. C. Preparation and Characterization of Polymeric Nanoparticles: An Interdisciplinary Experiment. J. Chem. Educ. 2016, 93 (8), 14461451.

(2) Lee, N.; Choi, Y.; Lee, Y.; Park, M.; Moon, W. K.; Choi, S. H.; Hyeon, T. WaterDispersible Ferrimagnetic Iron Oxide Nanocubes with Extremely High r 2 Relaxivity for Highly Sensitive in Vivo MRI of Tumors. Nano Lett. 2012, 12 (6), 3127-3131.

(3) Bjerle, P. Tension, Stress and Modulus of Elasticity of the Urinary Bladder Wall. Acta Physiol. Scand. 1974, 92 (4), 488-495.

(4) Yang, B.; Song, J.; Jiang, Y.; Li, M.; Wei, J.; Qin, J.; Peng, W.; Lasaosa, F. L.; He, Y.; Mao, H.; Yang, J.; Gu, Z. Injectable Adhesive Self-Healing Multicross-Linked DoubleNetwork Hydrogel Facilitates Full-Thickness Skin Wound Healing. ACS Appl. Mater. Interfaces 2020, 12 (52), 57782-57797.

(5) Hong, S. H.; Kim, S.; Park, J. P.; Shin, M.; Kim, K.; Ryu, J. H.; Lee, H. Dynamic Bonds between Boronic Acid and Alginate: Hydrogels with Stretchable, Self-Healing, StimuliResponsive, Remoldable, and Adhesive Properties. Biomacromolecules 2018, 19 (6), 2053-2061.

(6) Yang, R.; Liu, X.; Ren, Y.; Xue, W.; Liu, S.; Wang, P.; Zhao, M.; Xu, H.; Chi, B. Injectable Adaptive Self-Healing Hyaluronic Acid/Poly ( $\gamma$-Glutamic Acid) Hydrogel for Cutaneous Wound Healing. Acta Biomater. 2021, 127 (30), 102-115.

(7) Qu, J.; Zhao, X.; Liang, Y.; Zhang, T.; Ma, P. X.; Guo, B. Antibacterial Adhesive Injectable Hydrogels with Rapid Self-Healing, Extensibility and Compressibility as Wound Dressing for Joints Skin Wound Healing. Biomaterials 2018, 183 (August), 185-199. 
(8) Kim, M. H.; Lee, J. N.; Lee, J.; Lee, H.; Park, W. H. Enzymatically Cross-Linked Poly $(\gamma-$ Glutamic Acid) Hydrogel with Enhanced Tissue Adhesive Property. ACS Biomater. Sci. Eng. 2020, 6 (5), 3103-3113.

(9) Wu, T.; Cui, C.; Huang, Y.; Liu, Y.; Fan, C.; Han, X.; Yang, Y.; Xu, Z.; Liu, B.; Fan, G.; Liu, W. Coadministration of an Adhesive Conductive Hydrogel Patch and an Injectable Hydrogel to Treat Myocardial Infarction. ACS Appl. Mater. Interfaces 2020, 12 (2), 2039-2048.

(10) Zhao, X.; Liang, Y.; Huang, Y.; He, J.; Han, Y.; Guo, B. Physical Double-Network Hydrogel Adhesives with Rapid Shape Adaptability, Fast Self-Healing, Antioxidant and NIR/PH Stimulus-Responsiveness for Multidrug-Resistant Bacterial Infection and Removable Wound Dressing. Adv. Funct. Mater. 2020, 30 (17), 1-18.

(11) Ye, M.; Jiang, R.; Zhao, J.; Zhang, J.; Yuan, X.; Yuan, X. In Situ Formation of Adhesive Hydrogels Based on PL with Laterally Grafted Catechol Groups and Their Bonding Efficacy to Wet Organic Substrates. J. Mater. Sci. Mater. Med. 2015, 26 (12), 1-13.

(12) Yuan, L.; Wu, Y.; Fang, J.; Wei, X.; Gu, Q.; El-Hamshary, H.; Al-Deyab, S. S.; Morsi, Y.; Mo, X. Modified Alginate and Gelatin Cross-Linked Hydrogels for Soft Tissue Adhesive. Artif. Cells, Nanomedicine Biotechnol. 2017, 45 (1), 76-83.

(13) Sun, W.; Duan, T.; Cao, Y.; Li, H. An Injectable Self-Healing Protein Hydrogel with Multiple Dissipation Modes and Tunable Dynamic Response. Biomacromolecules 2019, $20(11), 4199-4207$.

(14) Chen, K.; Feng, Y.; Zhang, Y.; Yu, L.; Hao, X.; Shao, F.; Dou, Z.; An, C.; Zhuang, Z.; Luo, Y.; Wang, Y.; Wu, J.; Ji, P.; Chen, T.; Wang, H. Entanglement-Driven Adhesion, Self-Healing, and High Stretchability of Double-Network PEG-Based Hydrogels. ACS Appl. Mater. Interfaces 2019, 11 (40), 36458-36468. 
(15) Wang, L.; Zhang, X.; Yang, K.; Fu, Y. V.; Xu, T.; Li, S.; Zhang, D.; Wang, L. N.; Lee, C. S. A Novel Double-Crosslinking-Double-Network Design for Injectable Hydrogels with Enhanced Tissue Adhesion and Antibacterial Capability for Wound Treatment. Adv. Funct. Mater. 2020, 30 (1), 1-14.

(16) Cui, C.; Wu, T.; Gao, F.; Fan, C.; Xu, Z.; Wang, H.; Liu, B.; Liu, W. An Autolytic High Strength Instant Adhesive Hydrogel for Emergency Self-Rescue. Adv. Funct. Mater. 2018, 28 (42), 1-12.

(17) Zhao, X.; Wu, H.; Guo, B.; Dong, R.; Qiu, Y.; Ma, P. X. Antibacterial Anti-Oxidant Electroactive Injectable Hydrogel as Self-Healing Wound Dressing with Hemostasis and Adhesiveness for Cutaneous Wound Healing. Biomaterials 2017, 122, 34-47.

(18) Hu, W.; Zhang, Z.; Lu, S.; Zhang, T.; Zhou, N.; Ren, P.; Wang, F.; Yang, Y.; Ji, Z. Assembled Anti-Adhesion Polypropylene Mesh with Self-Fixable and Degradable: In Situ Mussel-Inspired Hydrogel Coating for Abdominal Wall Defect Repair. Biomater. Sci. 2018, 6 (11), 3030-3041.

(19) Deng, J.; Tang, Y.; Zhang, Q.; Wang, C.; Liao, M.; Ji, P.; Song, J.; Luo, G.; Chen, L.; Ran, X.; Wei, Z.; Zheng, L.; Dang, R.; Liu, X.; Zhang, H.; Zhang, Y. S.; Zhang, X.; Tan, H. A Bioinspired Medical Adhesive Derived from Skin Secretion of Andrias Davidianus for Wound Healing. Adv. Funct. Mater. 2019, 29 (31), 1-13.

(20) Han, L.; Lu, X.; Liu, K.; Wang, K.; Fang, L.; Weng, L. T.; Zhang, H.; Tang, Y.; Ren, F.; Zhao, C.; Sun, G.; Liang, R.; Li, Z. Mussel-Inspired Adhesive and Tough Hydrogel Based on Nanoclay Confined Dopamine Polymerization. ACS Nano 2017, 11 (3), 25612574.

(21) Heichel, D. L.; Burke, K. A. Dual-Mode Cross-Linking Enhances Adhesion of Silk Fibroin Hydrogels to Intestinal Tissue. ACS Biomater. Sci. Eng. 2019, 5 (7), 3246-3259. 
(22) Lalevée, G.; David, L.; Montembault, A.; Blanchard, K.; Meadows, J.; Malaise, S.; Crépet, A.; Grillo, I.; Morfin, I.; Delair, T.; Sudre, G. Highly Stretchable Hydrogels from Complex Coacervation of Natural Polyelectrolytes. Soft Matter 2017, 13 (37), 65946605 .

(23) Desai, M. S.; Chen, M.; Hong, F. H. J.; Lee, J. H.; Wu, Y.; Lee, S. W. CatecholFunctionalized Elastin-like Polypeptides as Tissue Adhesives. Biomacromolecules 2020, 21 (7), 2938-2948.

(24) Puertas-Bartolomé, M.; Benito-Garzón, L.; Fung, S.; Kohn, J.; Vázquez-Lasa, B.; San Román, J. Bioadhesive Functional Hydrogels: Controlled Release of Catechol Species with Antioxidant and Antiinflammatory Behavior. Mater. Sci. Eng. C 2019, 105 (July), 110040.

(25) Qu, J.; Zhao, X.; Liang, Y.; Zhang, T.; Ma, P. X.; Guo, B. Antibacterial Adhesive Injectable Hydrogels with Rapid Self-Healing, Extensibility and Compressibility as Wound Dressing for Joints Skin Wound Healing. Biomaterials 2018, 183 (August), $185-199$.

(26) Yang, Y.; Zhang, J.; Liu, Z.; Lin, Q.; Liu, X.; Bao, C.; Wang, Y.; Zhu, L. TissueIntegratable and Biocompatible Photogelation by the Imine Crosslinking Reaction. $A d v$. Mater. 2016, 28 (14), 2724-2730.

(27) Qiao, Z.; Mieles, M.; Ji, H. F. Injectable and Moldable Hydrogels for Use in Sensitive and Wide Range Strain Sensing Applications. Biopolymers 2020, 111 (6), 1-11.

(28) Feng, Q.; Wei, K.; Lin, S.; Xu, Z.; Sun, Y.; Shi, P.; Li, G.; Bian, L. Mechanically Resilient, Injectable, and Bioadhesive Supramolecular Gelatin Hydrogels Crosslinked by Weak Host-Guest Interactions Assist Cell Infiltration and in Situ Tissue Regeneration. Biomaterials 2016, 101, 217-228. 
(29) Gowda, A. H. J.; Bu, Y.; Kudina, O.; Krishna, K. V.; Bohara, R. A.; Eglin, D.; Pandit, A. Design of Tunable Gelatin-Dopamine Based Bioadhesives. Int. J. Biol. Macromol. 2020, 164, 1384-1391.

(30) Wang, J.; Zhang, F.; Tsang, W. P.; Wan, C.; Wu, C. Fabrication of Injectable High Strength Hydrogel Based on 4-Arm Star PEG for Cartilage Tissue Engineering. Biomaterials 2017, 120, 11-21.

(31) Lei, N.; Gong, C.; Qian, Z.; Luo, F.; Wang, C.; Wang, H.; Wei, Y. Therapeutic Application of Injectable Thermosensitive Hydrogel in Preventing Local Breast Cancer Recurrence and Improving Incision Wound Healing in a Mouse Model. Nanoscale 2012, $4(18), 5686-5693$.

(32) Xu, X.; Gu, Z.; Chen, X.; Shi, C.; Liu, C.; Liu, M.; Wang, L.; Sun, M.; Zhang, K.; Liu, Q.; Shen, Y.; Lin, C.; Yang, B.; Sun, H. An Injectable and Thermosensitive Hydrogel: Promoting Periodontal Regeneration by Controlled-Release of Aspirin and Erythropoietin. Acta Biomater. 2019, 86, 235-246.

(33) Jing, X.; Mi, H. Y.; Lin, Y. J.; Enriquez, E.; Peng, X. F.; Turng, L. S. Highly Stretchable and Biocompatible Strain Sensors Based on Mussel-Inspired Super-Adhesive SelfHealing Hydrogels for Human Motion Monitoring. ACS Appl. Mater. Interfaces 2018, 10 (24), 20897-20909.

(34) Wei, K.; Chen, X.; Zhao, P.; Feng, Q.; Yang, B.; Li, R.; Zhang, Z. Y.; Bian, L. Stretchable and Bioadhesive Supramolecular Hydrogels Activated by a One-StoneTwo-Bird Postgelation Functionalization Method. ACS Appl. Mater. Interfaces 2019, 11 (18), 16328-16335.

(35) Zhang, H.; Bré, L. P.; Zhao, T.; Zheng, Y.; Newland, B.; Wang, W. Mussel-Inspired Hyperbranched Poly(Amino Ester) Polymer as Strong Wet Tissue Adhesive. Biomaterials 2014, 35 (2), 711-719. 
(36) Zhang, H.; Zhao, T.; Duffy, P.; Dong, Y.; Annaidh, A. N.; O’Cearbhaill, E.; Wang, W. Hydrolytically Degradable Hyperbranched PEG-Polyester Adhesive with Low Swelling and Robust Mechanical Properties. Adv. Healthc. Mater. 2015, 4 (15), 2260-2268.

(37) Burke, S. A.; Ritter-Jones, M.; Lee, B. P.; Messersmith, P. B. Thermal Gelation and Tissue Adhesion of Biomimetic Hydrogels. Biomed. Mater. 2007, 2 (4), 203-210.

(38) Chung, H.; Grubbs, R. H. Rapidly Cross-Linkable DOPA Containing Terpolymer Adhesives and PEG-Based Cross-Linkers for Biomedical Applications. Macromolecules 2012, 45 (24), 9666-9673.

(39) Krishnadoss, V.; Melillo, A.; Kanjilal, B.; Hannah, T.; Ellis, E.; Kapetanakis, A.; Hazelton, J.; San Roman, J.; Masoumi, A.; Leijten, J.; Noshadi, I. Bioionic Liquid Conjugation as Universal Approach to Engineer Hemostatic Bioadhesives. ACS Appl. Mater. Interfaces 2019, 11 (42), 38373-38384.

(40) Pinkas, O.; Goder, D.; Noyvirt, R.; Peleg, S.; Kahlon, M.; Zilberman, M. Structuring of Composite Hydrogel Bioadhesives and Its Effect on Properties and Bonding Mechanism. Acta Biomater. 2017, 51, 125-137.

(41) Sun, J. Y.; Zhao, X.; Illeperuma, W. R. K.; Chaudhuri, O.; Oh, K. H.; Mooney, D. J.; Vlassak, J. J.; Suo, Z. Highly Stretchable and Tough Hydrogels. Nature 2012, 489 (7414), 133-136.

(42) Noshadi, I.; Hong, S.; Sullivan, K. E.; Shirzaei Sani, E.; Portillo-Lara, R.; Tamayol, A.; Shin, S. R.; Gao, A. E.; Stoppel, W. L.; Black, L. D.; Khademhosseini, A.; Annabi, N. In Vitro and in Vivo Analysis of Visible Light Crosslinkable Gelatin Methacryloyl (GelMA) Hydrogels. Biomater. Sci. 2017, 5 (10), 2093-2105.

(43) Fan, H.; Wang, J.; Zhang, Q.; Jin, Z. Tannic Acid-Based Multifunctional Hydrogels with Facile Adjustable Adhesion and Cohesion Contributed by Polyphenol Supramolecular Chemistry. ACS Omega 2017, 2 (10), 6668-6676. 
(44) Koushki, N.; Katbab, A. A.; Tavassoli, H.; Jahanbakhsh, A.; Majidi, M.; Bonakdar, S. A New Injectable Biphasic Hydrogel Based on Partially Hydrolyzed Polyacrylamide and Nanohydroxyapatite as Scaffold for Osteochondral Regeneration. RSC Adv. 2015, 5 (12), 9089-9096.

(45) Li, J.; Celiz, A. D.; Yang, J.; Yang, Q.; Wamala, I.; Whyte, W.; Seo, B. R.; Vasilyev, N. V.; Vlassak, J. J.; Suo, Z.; Mooney, D. J. Tough Adhesives for Diverse Wet Surfaces. Science 2017, 357 (6349), 378-381. 